\title{
Deficits of visual working memory representations of emotional facial expressions in patients with congenital facial palsy
}

\author{
Filippo Gambarota ${ }^{1}$, Roy Luria ${ }^{2,3}$, Massimiliano Pastore $^{1}$, Elisa De Stefani ${ }^{4}$, Pier \\ Francesco Ferrari ${ }^{* *}$, \& Paola Sessa ${ }^{1,6^{*}}$
}

${ }^{1}$ Department of Developmental and Social Psychology, University of Padova, Padova, Italy

${ }^{2}$ The School of Psychological Sciences, Tel Aviv University, Tel Aviv, Israel

${ }^{3}$ Sagol School of Neuroscience, Tel Aviv University, Tel Aviv, Israel

${ }^{4}$ Department of Medicine and Surgery, University of Parma, Parma, Italy

${ }^{5}$ Institut des Sciences Cognitives Marc Jeannerod, CNRS/Université Claude Bernard Lyon, France

${ }^{6}$ Padova Neuroscience Center (PNC), University of Padova, Padova, Italy

The dataset and analysis reported in this manuscript are available at this Open Science Framework repository: $\underline{\text { osf.io/4QXVG }}$

\section{*Co-Corresponding authors:}

Paola Sessa, Department of Developmental and Social Psychology, University of Padova, Via Venezia 8, 35121, Padua, Italy.

paola.sessa@unipd.it

Pier Francesco Ferrari, Institut des Sciences Cognitives Marc Jeannerod, Unit 5229, CNRS/ Université Claude Bernard Lyon, 67 Bd Pinel, 69675 Bron Cedex, France

pierfrancesco.ferrari@isc.cnrs.fr

Key words: visual working memory, delayed estimation task, motor simulation, facial expressions, Moebius syndrome 


\section{words count $=\mathbf{5 1 8 3}$}

\section{Abstract}

Recent models of sensorimotor simulation postulate that simulation aids 'refining' visual representations of others' emotional facial expressions through an iterative communication with the visual system. In order to test this aspect of sensorimotor simulation models, in the present investigation we recruited a sample of patients with Moebius syndrome (MS), characterized by congenital facial paralysis, and a control group of healthy participants. MS patients constitute a particularly interesting test as their congenital impossibility of producing facial movements and expressing emotions through the face should translate into a deficit of facial expressions processing. Here, MS and healthy participants underwent a task aimed at measuring the precision with which representations of facial expressions of emotions are maintained in visual working memory (VWM) by implementing a delayed estimation task. In each trial, participants saw a face with a certain intensity of facial expression (i.e., of happiness, disgust or anger) and, after a short time interval of about $1 \mathrm{sec}$, they had to select the to-bememorized image inside a circular array in which facial expressions of increasing intensity were presented in an orderly manner. The results indicated that MS participants built lower quality representations of the intensity of emotional expressions when compared to healthy participants. These findings support the role of sensorimotor simulation in improving the quality of emotional representations of facial expressions during early stages of processing. 


\section{Introduction}

Humans are expert 'face processors'. A large body of evidence indicates that faces are subject to analysis by a functional (McKone et al., 2011) and neural (Kanwisher et al., 1997; Haxby et al., 2000; Kanwisher and Yovel, 2006) architecture responsible for their processing. Faces are an extremely rich source of socio-affective information such as somatic traits, race and emotions expressed through the activation of specific facial muscle patterns (Adolphs, 2002; Adolphs and Birmingham, 2011). These muscular activation patterns produce what are called 'facial expressions' whose evolutionary valence had already been recognized by Charles Darwin (Darwin, 1872; Ekman, 2003).

Several theoretical models have been proposed to characterize human expertise in processing facial expressions and in assigning a meaning in terms of emotional states to these facial expressions. In at least partial opposition to an explanation in terms of the visualcognitive mechanism (Bruce and Young, 1986), these so-called simulation models hypothesize that internal re-enactment of a motor program associated with the observed expression and an internal reproduction of the associated emotional state underlie efficient emotion processing and recognition (Goldman and Sripada, 2005). Although several nuanced simulation models can be counted (for a review see Goldman and Sripada, 2005), a common aspect regards the fast and automatic activation of coherent sensorimotor representations according to the observed emotional facial expression. This process can also be supported by facial mimicry, namely the rapid and subtle activation of facial muscles involved in the observed expression (Dimberg, 1982; Dimberg et al., 2000; Du et al., 2016). A recent theoretical model by Wood and colleagues (2016) integrates sensorimotor, peripheral (i.e. facial mimicry) and cognitive (e.g. previous knowledge and contextual information) processing as all fundamental components for emotions recognition. 
One important prediction of these simulation models, including that by Wood and colleagues (2016), is that interfering with the simulation process, such as blocking facial mimicry, should impair facial emotions recognition/discrimination (Oberman et al., 2007; Stel and van Knippenberg, 2008; Ponari et al., 2012; Ipser and Cook, 2015; Davis et al., 2017; Borgomaneri et al., 2020) especially in tasks designed to assess the perceptual, rather than the verbal, component of expression recognition. For the aim of the present investigation, it is important to note that a critical facet of Wood and colleagues' (2016) model regards the bidirectional iterative communication between visual and sensorimotor regions such that the quality of the visual percepts of facial expressions can be modulated by the sensorimotor activity which would support an effective emotion recognition. In fact, blocking the facial mimicry seems not only to interfere with the ability to verbally recognize facial expressions (Oberman et al., 2007; see Rychlowska et al., 2014 for smiles detection; Wood, Lupyan, et al., 2016) but also with the quality of visual working memory (VWM) representations (Sessa et al., 2018). In Sessa and colleagues' study (2018), a standard change detection task (CDT) was implemented with neutral, subtle and full facial expressions of anger as to-be-remembered stimuli. Accuracy score in CDT, empathic self-report scores and a specific event-related potential (ERP) tagging VWM, named Sustained Posterior Contralateral Negativity (SPCN), were collected. Previous research showed how this ERP component reflects both the quantity and quality of stimuli represented in VWM (Vogel and Machizawa, 2004; Jolicœur et al., 2011; Sessa et al., 2011; Sessa et al., 2012; Meconi et al., 2014; for a review see Luria et al., 2016; Sessa and Dalmaso, 2016; Sessa et al., 2018; Gambarota and Sessa, 2019). Importantly, the study by Sessa and colleagues (2018) included a crucial within-subjects manipulation of participants' facial mimicry that was altered/blocked by using a hardener mask in one critical condition. Results showed that neutral and full facial expressions were associated with better performance and greater SPCN amplitude compared to subtle expressions. Notably, blocking 
facial mimicry led to a decrease of SPCN amplitude (i.e. lower-quality VWM representations) especially as a function of participants' empathy scores assessed by means of Empathy Quotient questionnaire (Baron-Cohen and Wheelwright, 2004). These findings are particularly intriguing when considering that VWM is a core cognitive system that retains visual information for further processing and it is considered a critical hub between low (e.g., perceptual stages) and high level processing (e.g., decision making, long-term memory, cf., (Luck, 2008).

In light of these premises, it is clear that clinical conditions in which the possibility of using facial mimicry is precluded represents an extremely relevant 'source' for testing the sensorimotor simulation model. In particular, the central question of the present study was whether the quality of the VWM representations of facial expressions of emotions might be compromised in subjects with impaired facial mimicry such, for instance, in neurological diseases like Parkinson (e.g. Gray and Tickle-Degnen, 2010 for a meta-analysis) or in individuals who have bilateral congenital facial palsy (for a review see De Stefani et al., 2019) such as in Moebius syndrome patients. These last patients, in addition to presenting an impairment of facial mimicry, could be strongly deficient in sensorimotor 'vocabulary' of facial expressions of emotions.

The main aim of the present study was to investigate the contribution of the sensorimotor system to the processing of facial expressions. For this purpose, we considered a group of participants with Moebius syndrome (Kumar, 1990; Pedersen et al., 2017), namely a rare and congenital condition of which one of the main manifestations is a facial paralysis which precludes the possibility of expressing one's emotional states through the facial musculature. The question the present study poses addressed one of the mechanisms suggested as crucial for the processing of facial expressions of emotions, i.e. sensorimotor re-enactment of the observed expressions. 
We recruited patients with Moebius Syndrome. A correct diagnosis is challenging due to unclear criteria (Picciolini et al., 2016) and the prevalence is estimated to be 1 in 50.000 to 1 in 500.000 live births (Lindsay et al., 2010; Rasmussen et al., 2015; Picciolini et al., 2016; De Stefani et al., 2019). Within this complex syndromic framework, the main clinical aspect is the partial or total facial palsy caused by a congenital malformation of the VI (abducent) and VII (facial) cranial nerves (Kumar, 1990; De Stefani et al., 2019). Despite the inability to produce facial expressions, these patients seem to have a normal cognitive development (De Stefani et al., 2019).

Literature on psychosocial functioning, and in particular about facial expression processing, is sparse and unclear, probably for methodological reasons and rarity of the condition, and mostly focused on the verbal component of emotion recognition (i.e., 'labeling' tasks). Giannini and colleagues (1984) and Bate and colleagues (2013), using an emotion identification task from facial stimuli, found a mild deficit in Moebius patients. Using a similar approach, no deficits were reported by Calder et al. (2000) and Bogart and Matsumoto (2010). Importantly, sample size ranged from 1 subject (Giannini et al., 1984) to 37 patients (Bogart and Matsumoto, 2010). However, in the latter study the recruiting procedure was partially based on a self-report diagnosis and the experimental procedure included only full emotional expressions. Interestingly, recording also the autonomic response in children with Moebius Syndrome, Nicolini and colleagues (2019) showed an emotion recognition deficit and a reduced autonomic response to emotional stimuli. Except for Nicolini and colleagues' (2019) study, all previous investigations mainly focused on the verbal component of emotion recognition.

Several studies demonstrated the relevance of the VWM system in social cognition, in particular for facial stimuli (for a review see Gambarota and Sessa, 2019). Among several experimental paradigms to study VWM, the Delayed Estimation Task (DET) (Zhang and Luck, 
2008) is well suited to measure the precision of visual representations and, importantly, previous studies have implemented variants of the DET with faces as stimuli (Krill et al., 2018; Yao et al., 2018). The general structure of a typical trial consists of a sequence of two stimulus arrays, interleaved by a temporal delay, in which the first is a single to-be-remembered stimulus and the second a test array of stimuli physically similar to the single to-be-remembered stimulus. Participants are required to compare the representation of the first stimulus with the stimuli in the test array, selecting the stimulus matching the to-be-remembered stimulus. A distribution of memory performance can be obtained computing the difference (e.g. according to the manipulated stimulus feature such as the facial expression intensity) between the to-beremembered stimulus and the selected one. As reported before, to understand the impact of sensorimotor simulation on emotion recognition in general, and on VWM representations of facial expressions in particular, external facial mimicry manipulations (e.g using a hardening face mask) have been usually adopted. This procedure is straightforward to apply but could lead to measurement noise and variability in results. Most importantly, blocking and/or altering facial mimicry has an indirect effect on sensorimotor simulation, since it provides an incongruent feedback with the re-enactment of the observed facial expression. Congenital conditions of facial paralysis, except for possible compensation mechanisms during the course of development, therefore appear particularly relevant in this context of studies. The sensorimotor deficit could be particularly evident when the task focuses on visual processing, as suggested by the iterative mechanism between sensorimotor and visual brain regions postulated by Wood and colleagues' (2016) model.

In light of these premises, in the present study we aimed at using DET to estimate VWM precision for emotional facial expressions in Moebius participants compared to a control group of healthy participants. Importantly, the DET paradigm - and the VWM in general - is strictly related to visual processing, in particular in this context to the quality of VWM representations 
of facial expressions, and no explicit verbal response is required. We hypothesized lower VWM precision - indexed by both higher mean and higher variability of VWM performance distribution (i.e., higher number of errors) - in Moebius participants compared to control participants. Additionally, we performed an exploratory analysis comparing VWM performance for different facial expressions of emotions (i.e. anger, happiness and fear) given that the role of sensorimotor simulation/facial mimicry for different facial expressions is matter of current debate (for a review see Hess and Fischer, 2014).

\section{Methods}

\subsection{Participants}

Participants were a group of 7 patients ( 3 females) with a certified diagnosis of Moebius syndrome and a mean age of 34 years $(\mathrm{SD}=10.5)$. A group of 30 volunteers ( 15 females) with a mean age of $24.2(\mathrm{SD}=4.6)$ was recruited as healthy controls. All participants reported normal or corrected-to-normal vision. Sample size for the Moebius group is considerable compared to previous studies and when considering the rarity of this congenital condition (Calder et al., 2000; for a review see also De Stefani et al., 2019). In Table 1 are reported demographic data and clinical information concerning all participants with Moebius syndrome. All patients have a certified diagnosis of Moebius syndrome. The sample size for the control group was chosen based on a previous similar study (Krill et al., 2018). Given the complexity of the statistical model (see Data analysis section) a comparison between the Moebius and normative population was preferred instead of a one by one matching. 
VWM deficits in Moebius subjects - Gambarota et al.

Table 1 Descriptive statistics of Memory Errors and Reaction Times

\begin{tabular}{|c|c|c|c|c|c|}
\hline Subject & Age & Gender & Paralysis & Cranial nerves involved & Dysfunction \\
\hline \multirow{2}{*}{ MS1 } & \multirow{2}{*}{37} & \multirow{2}{*}{ Male } & \multirow{2}{*}{ Bilateral } & Abducens Nerve (VI) & No lateral eye movements \\
\hline & & & & Facial Nerve (VII) & Facial palsy \\
\hline \multirow{2}{*}{ MS2 } & \multirow{2}{*}{22} & \multirow{2}{*}{ Female } & \multirow{2}{*}{ Bilateral } & Abducens Nerve (VI) & No lateral eye movements \\
\hline & & & & Facial Nerve (VII) & Facial palsy \\
\hline \multirow{2}{*}{ MS3 } & \multirow{2}{*}{20} & \multirow{2}{*}{ Male } & \multirow{2}{*}{ Bilateral } & Abducens Nerve (VI) & No lateral eye movements \\
\hline & & & & Facial Nerve (VII) & Facial palsy \\
\hline \multirow{2}{*}{ MS4 } & \multirow{2}{*}{35} & \multirow{2}{*}{ Male } & \multirow{2}{*}{ Bilateral } & Abducens Nerve (VI) & No lateral eye movements \\
\hline & & & & Facial Nerve (VII) & Facial palsy \\
\hline MS5 & 33 & Female & $\begin{array}{c}\text { Monolateral } \\
\text { (left) }\end{array}$ & Facial Nerve (VII) & Facial Palsy \\
\hline \multirow{2}{*}{ MS6 } & \multirow{2}{*}{54} & \multirow{2}{*}{ Male } & \multirow{2}{*}{ Bilateral } & Abducens Nerve (VI) & No lateral eye movements \\
\hline & & & & Facial Nerve (VII) & Facial palsy \\
\hline \multirow{2}{*}{ MS7 } & \multirow{2}{*}{37} & \multirow{2}{*}{ Female } & \multirow{2}{*}{ Bilateral } & Abducens Nerve (VI) & No lateral eye movements \\
\hline & & & & Facial Nerve (VII) & Facial palsy \\
\hline
\end{tabular}

\subsection{Stimuli}

Facial expression stimuli were selected from the MMI Facial Expression Database (Pantic et al., 2005). Three emotional videos of two different participants (one female and one male) expressing anger, happiness and fear were segmented frame by frame according to the videospecific frame rate (25 frames per second). A total of 8 images for each emotion and identity were selected (48 total images). The first and the last images were respectively the neutral (before the facial expression onset) and the facial expression maximum peak, obtaining an ascending scale of facial expression (from 0 to 7; see Figure 1B for an example of stimuli). Given that different facial expressions seem to have different movement kinematics (Ekman \& Friesen, 1975; Sato \& Yoshikawa, 2004) the frames between the neutral and the peak, were selected to be as different as possible from each other using a subjective criterion. Final images were, resized to subtend at $4.3^{\circ} \times 3.74^{\circ}$ visual angles (resolution of $157 \times 130$ pixels) 
and oval cropped to remove the background and hair. For visualization purposes, stimuli were greyscale converted, adjusted in luminance (reduction of 35\%) and displayed on a black background. Frames extraction and image manipulation was performed with the FFmpeg (Version 4.2.1), Matlab (version R2019a) and ImageMagick (version 6) software. All stimuli, paradigm and image processing scripts are available in the online repository (ㅇf.io/4QXVG).

\subsection{Procedure}

We used a Delayed Estimation Task (DET) (Krill et al., 2018; Ma et al., 2014; Yao et al., 2018; Zhang \& Luck, 2008) which is a variant of the change detection task (Luck \& Vogel, 1997; Rensink, 2002; Vogel et al., 2001). In general, in a standard DET a memory display with a single to-be-memorized item is briefly presented followed by a blank retention interval and a final test display. The participant has to compare the to-be-memorized item representation with a continuous array of items that differ in memory-test similarity (see Figure 1A for a trial example). The difference between the to-be-memorized item and the participant response create a distribution of memory errors that can be analysed in different ways as a function of stimulus features, in particular both the mean and the variability can be considered as indices of memory precision (but see Bays et al., 2009; see Krill et al., 2018; and Yao et al., 2018 for a different application with facial stimuli; and Zhang \& Luck, 2008 for a mathematical modelling of DET performance).

The experiment was programmed in EPrime 2.0 (Psychology Software Tools, Pittsburgh, PA) and took place in a dimly lit room. Participants were seated $50 \mathrm{~cm}$ from the screen of a 15.6-inch laptop monitor (resolution of 1366 x 768 pixels). Each trial began with a fixation cross for $500 \mathrm{~ms}$. Then a to-be-memorized face (Facial Expression Level), randomly selected from the set of stimuli (0 to 7) of the same emotion/identity, was centrally presented for $500 \mathrm{~ms}$, presentation time that is appropriate to encode a single complex stimulus (Curby \& 
Gauthier, 2007; but see Krill et al., 2018). After a blank retention interval of $1000 \mathrm{~ms}$, the test array was presented with the entire set of facial expression levels arranged on a circumference with a diameter subtending to $16^{\circ}$ visual angles. The order of the circular presentation was fixed (i.e., clockwise ordered from 0 to 7) but the circumference rotated trial by trial unpredictably. Each facial expression level appeared once in each of possible positions and also as to-bememorized item. Using the mouse, participants had to select the to-be-memorized image. There was not a feedback on the correctness of their response. A blank intertrial display was presented for $1500 \mathrm{~ms}$. The test display lasted for 10 seconds and only accuracy was stressed. Before the experimental session, participants performed 8 training trials with faces stimuli not included in the experiment to become familiar with the procedure. In order to not tire the patients, one experimental block consisted of 48 trials (8 to-be-memorized Facial Expression Levels x 3 Emotions x 2 Identities) repeated twice for a total of 96 trials per participant. 
A

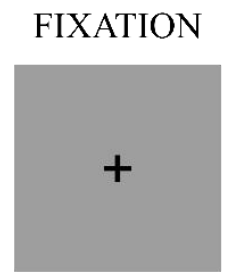

$500 \mathrm{~ms}$

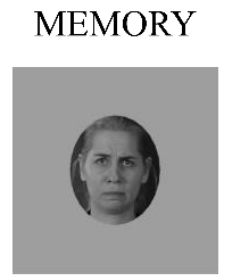

$500 \mathrm{~ms}$

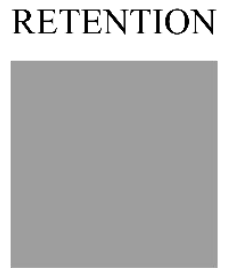

$1000 \mathrm{~ms}$
TEST

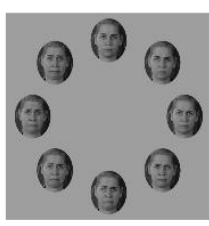

$10 \mathrm{~s}$
INTERTRIAL

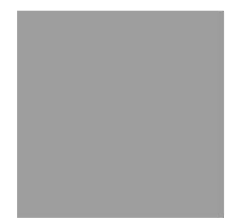

$1500 \mathrm{~ms}$

B

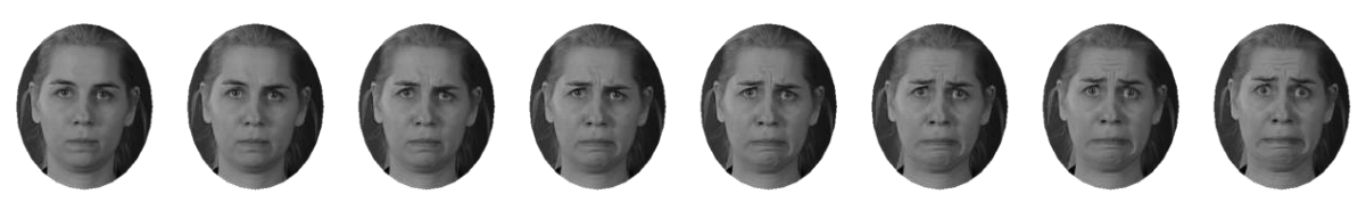

Figure 1 (A) Experimental paradigm. (B) Example array of experimental stimuli from neutral to full facial expression

\subsection{Data analysis}

The DET allows computing a quantitative distribution of performance. Our dependent variable (Memory Errors) was calculated as the difference between participant's response (test display; 0 to 7) and the Facial Expression Level (memory display; 0 to 7), such that the correct responses corresponded to 0 scores while the scores from 1 to 7 (in absolute values) represented an increasing error in visual memory representation. In such terms, a greater Memory Errors score represented lower memory precision. Levels of the identity factor (i.e., male faces and female faces) were collapsed in order to improve signal-to-noise ratio.

The error distribution (Memory Errors; in absolute values) was modeled using a linear mixed-effect model (LME) with Group (Moebius and Control participants), Emotion (Anger, Fear and Happiness) as factors and Facial Expression Level (to-be-memorized stimulus; 0 to 7) as covariate. Participants were considered as a random effect to deal with the repeated measure design. The Facial Expression Level is considered a quantitative variable given that no clear 
hypotheses were made according to the specific emotion intensity. Moreover, given the small sample size, the amount of trials and the continuous nature of the original stimulus (i.e. the emotional expression video) estimating a single parameter for each emotion (i.e. the linear changing of memory precision according to facial expression level) can be considered the most parsimonious choice in terms of model complexity and estimation precision for each parameter.

Besides the Memory Errors mean, also the variability of the distribution could be considered as an index of precision (see Yao et al., 2018). Instead of performing a separate model on the variance component, our analysis took into account the possible heterogeneity of variances among the Group variable (Pinheiro and Bates, 2000).

Starting from the full model (i.e the model that contains all interactions between predictors), a step-wise approach based on the Akaike Information Criteria (AIC) (Akaike, 1974) was used to select the best predictors combination that explained the data. Furthermore, using Akaike weights (Wagenmakers and Farrell, 2004) is possible to compare a set of alternative models in probabilistic terms. According to Nakagawa and colleagues $(2013 ; 2017)$, a coefficient of determination $\left(\mathrm{R}^{2}\right)$ was computed for the selected model. All further details in the result section refers to the best model. Reaction times (RTs) were inserted as a covariate to the selected Memory Errors model to adjust estimated parameters. Moreover, RTs were separately analysed with another linear mixed-effect model using Emotion and Group as predictors in order to validate results from the Memory Errors model. Crucially, given the amount of time available to respond (10 seconds) and the stressed focus on accuracy this analysis is considered completely exploratory. Analyses were performed using R software (R Core Team, 2020) and all scripts, data and used packages are available in the online repository (osf.io/4QXVG). 


\section{Results}

The frequency distribution of Memory Errors, grouped by displayed emotion, for Moebius patients and controls is depicted in figure 2. As an exploratory analysis the mean of each Facial Expression Level (i.e. considered as factor) is depicted in figure 3. In table 1 are reported means and standard deviations for Memory Errors score and RTs.

The selected regression model $\left(R^{2}=0.10\right.$ considering both fixed and random effects; $R^{2}$ $=0.07$ considering fixed effects only) contained the main effects of Group, Emotion and the interaction between Emotion and Facial Expression Level. In figure 4 are depicted all model effects. Notably, and in line with our main hypothesis, analysis of variance using the Satterthwaite's method for degrees of freedom approximation (Kuznetsova et al., 2017; Luke, $2017)$ showed a main effect of Group $\left(F_{(1,34.3)}=17, p=<0.001\right)$. Importantly, this indicated that Moebius patients showed a higher Memory Errors score compared to controls (Moebius Controls $\left.=0.38 ; \beta=0.40, \mathrm{SE}=0.09, t_{34}=4.12, p<0.001\right)$.

Emotion $\left(F_{(2,3843)}=10.61, p<0.001\right)$ and the interaction between Emotion and Facial Expression Level $\left(F_{(3,3843)}=11.19, p<0.001\right)$ were also significant. Moreover, also the effect of RTs was significant $\left(F_{(1,2839)}=73.23, p<0.001\right)$. The analysis of each emotion showed a significant relationship between Memory Errors and Facial Expression Level both for fearful $\left(\beta=0.04, S E=0.013, t_{3484}=3.35, p<0.001\right)$ and happy $\left(\beta=-0.04, S E=0.013, t_{3484}=-3.32, p\right.$ $=<0.001)$ faces. For angry faces the relationship was not significant $\left(\beta=0, S E=0.013, t_{3484}=\right.$ $-0.03, p=0.969$ ). Regarding RTs (in seconds), there was a positive relationship with Memory Errors (i.e. slower RTs for higher memory errors; $\beta=0.09, \mathrm{SE}=0.01, t_{2838}=8.55, \mathrm{p}<0.001$ ). A separate linear mixed-effect model on RTs revealed no effect main of Emotion and Group but an interaction between Emotion and Group. However, post-hoc comparisons for each emotion between Moebius e Controls showed no significant differences. For further details 
about models parameters, diagnostic and all exploratory analysis see the online repository (osf.io/4QXVG). As an exploratory analysis (since the best model did not contain the interaction between Group and Emotion), we also performed three planned comparisons for the interaction between Group and Emotion fitting a model with only these two predictors. All three emotions were associated with a higher Memory Errors score for the Moebius group $($ Control Anger - Moebius Anger $=-0.287, \mathrm{SE}=107$, t78.2-2.68, $p=0.027$; Control Fear Moebius Fear $=-0.477, \mathrm{SE}=107, t_{77.8}-4.45, p<0.001$; Control Happiness - Moebius Happiness $=-0.368, \mathrm{SE}=107, t_{78.2-3.434}, p=0.003 ; \mathrm{p}$-values were Bonferroni corrected).

In order to test the variability in Memory Errors between Moebius and control participants we fitted a model with and without the heteroscedastic component (For a detailed explanation of this method see Pinheiro and Bates, 2000) considering only the Group as predictor. The likelihood ratio test showed that the heteroscedastic model explains the data better than the non-heteroscedastic model $\left(\chi_{1}=126.75, p<0.001\right)$. In particular, the variance in Memory Errors for the control group is about $72 \%$ of Moebius' variance.

Table 2 Descriptive statistics of Memory Errors and Reaction Times

\begin{tabular}{cccc}
\hline Group & Emotion & Memory Errors & Reaction Times \\
\hline \multirow{3}{*}{ Controls } & Anger & $0.65(0.85)$ & $3519.84(1701.82)$ \\
& Fear & $1.13(1.10)$ & $3767.92(1886.13)$ \\
& Happiness & $0.68(0.87)$ & $3620.42(1623.98)$ \\
\hline \multirow{3}{*}{ Moebius Patients } & Anger & $0.94(1.12)$ & $3447.23(1562.93)$ \\
& Fear & $1.61(1.58)$ & $3311.69(1875.39)$ \\
& Happiness & $1.05(1.21)$ & $3442.36(1540.06)$ \\
\hline
\end{tabular}

Means and standard deviations (in brackets) for Memory Errors and Reaction Times for each Emotion and Group. 


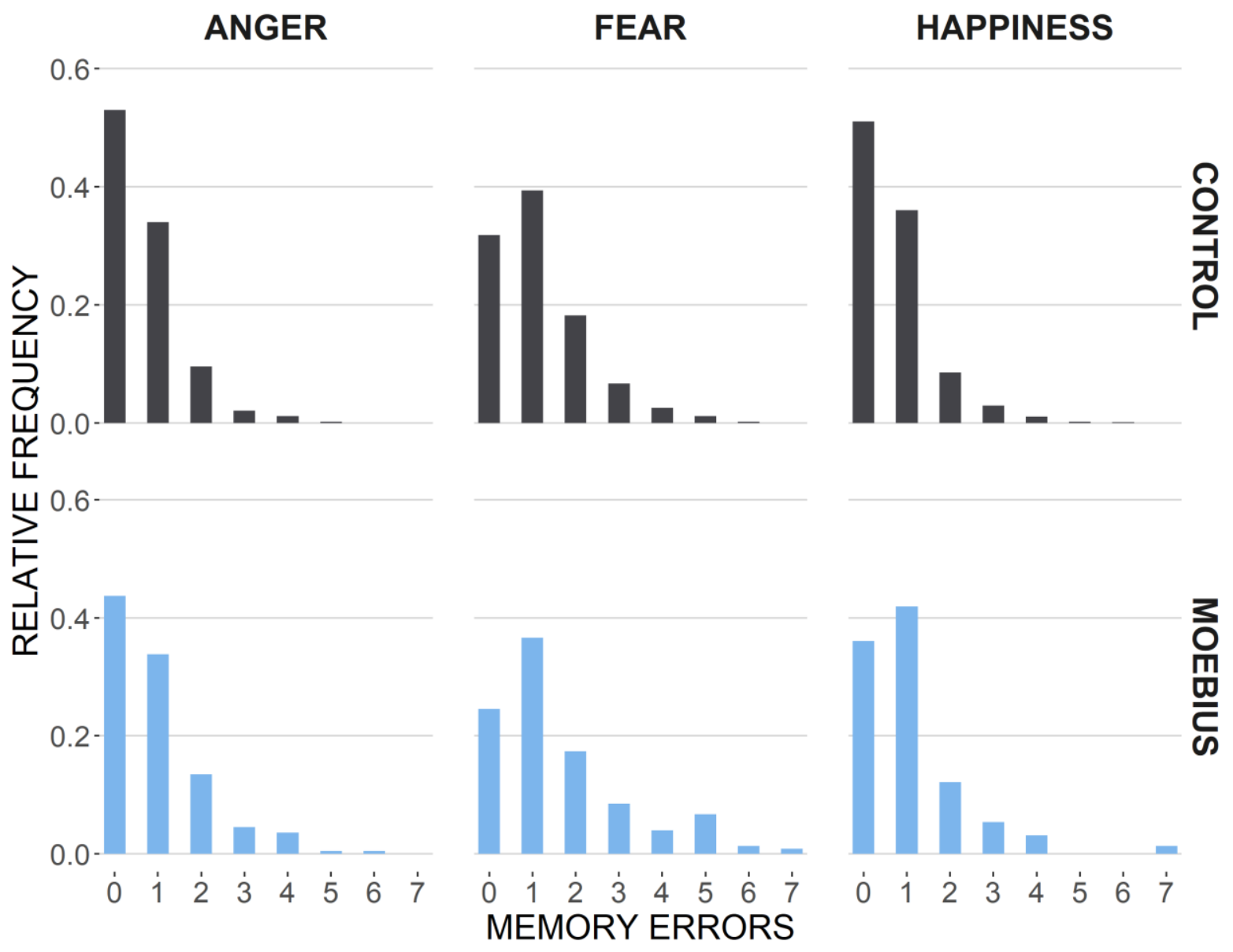

Figure 2 Distributions of memory errors (relative frequencies) according to displayed Emotion and Group.

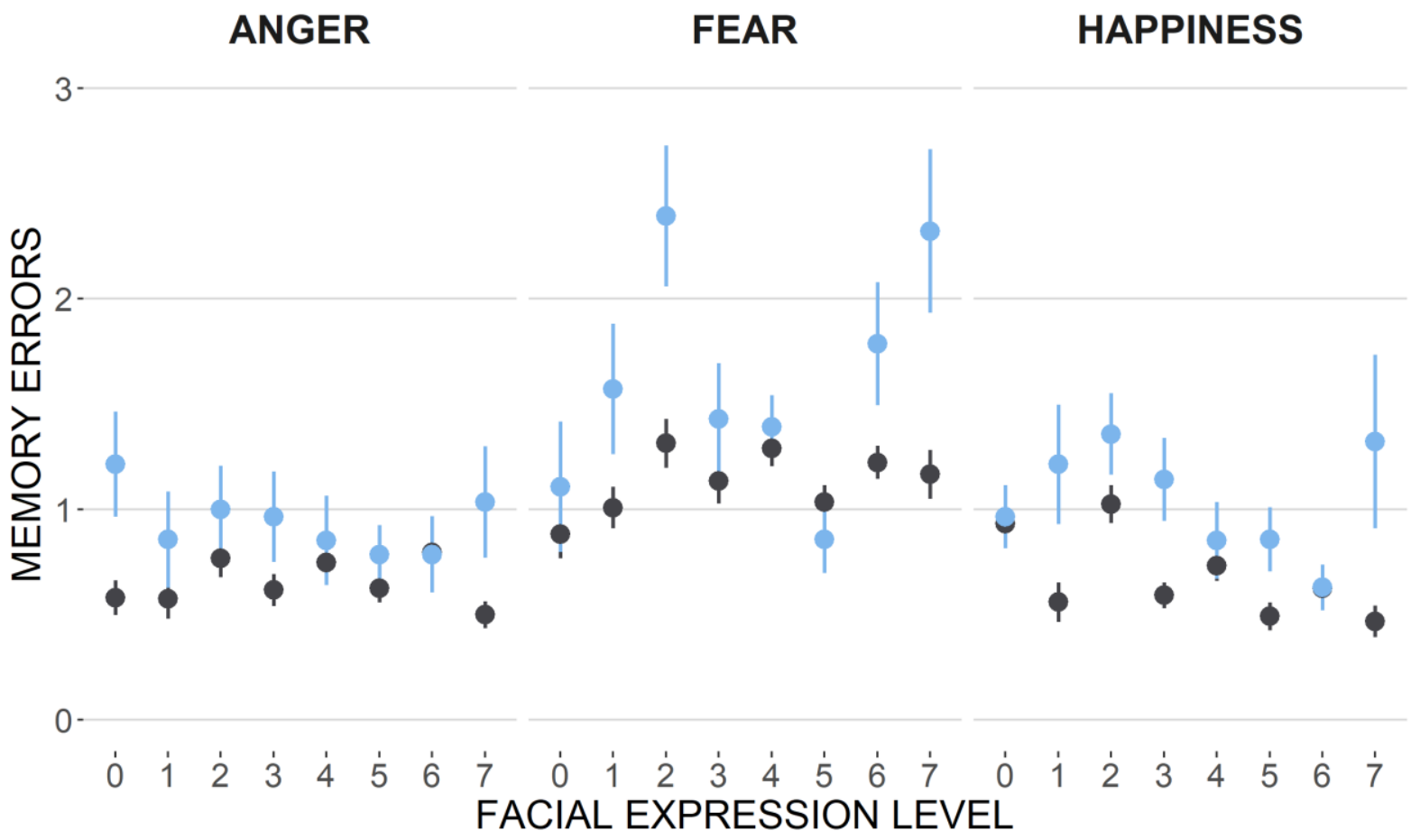

Figure 3 Means and standard errors of Memory Errors score according to displayed emotions, Group and Facial Expression Level. 


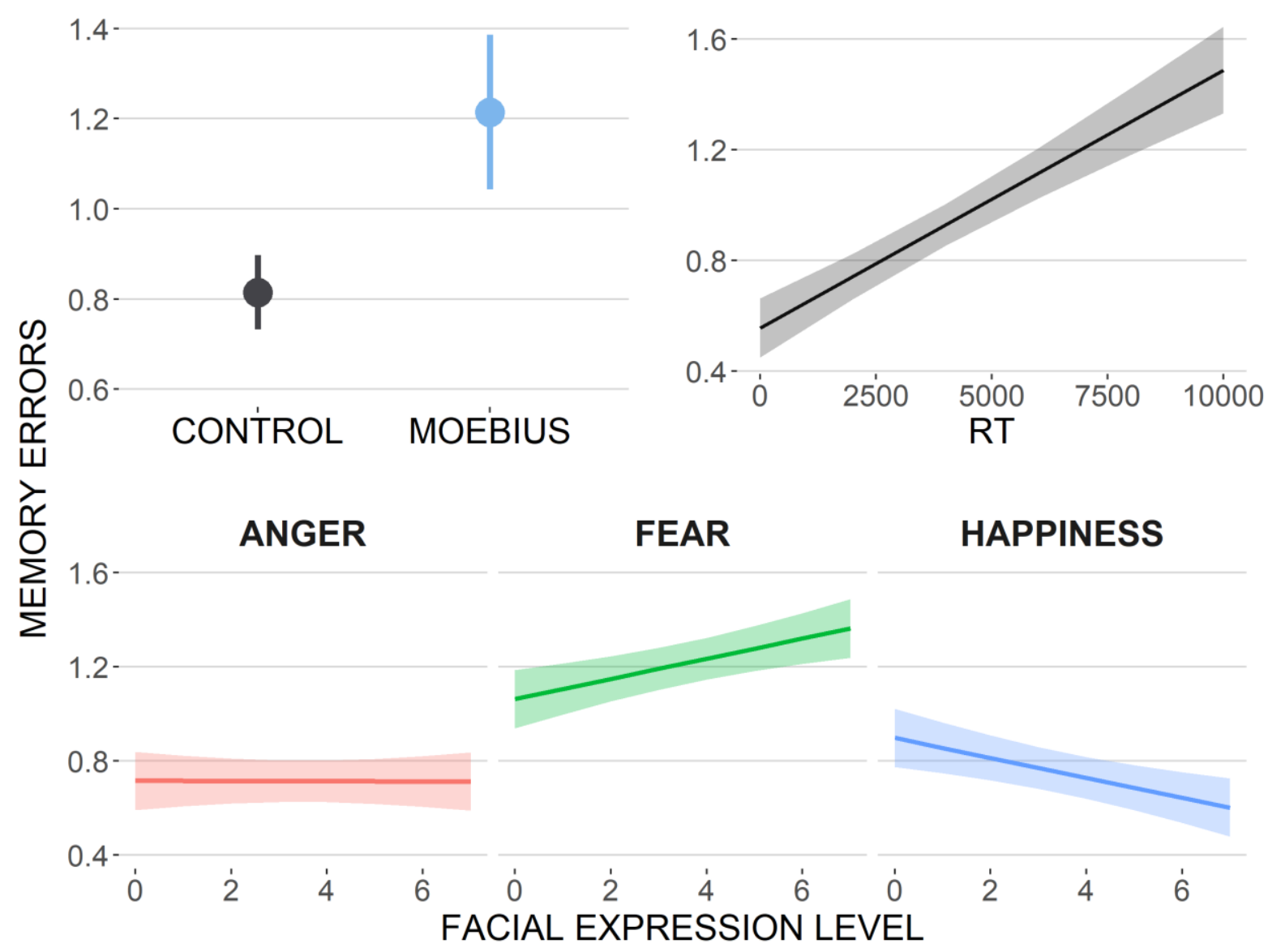

Figure 4 Estimated effects of the selected model. Shaded areas and bars represent the $95 \%$ confidence interval. Upper left: the main effect of Group. Points represent the estimated means. Upper right: the relationship between Reaction Times and Memory Errors. Below: Interaction between Facial Expression Level and displayed Emotion

\section{Discussion}

In the present study, the performance in a VWM task for facial expressions of seven patients with Moebius syndrome was compared with that of a control group of thirty healthy participants. The aim was to test sensorimotor simulation models and, more specifically, a facet of the sensorimotor simulation model by Wood and colleagues (2016), namely that the sensorimotor system iteratively communicates with the visual system during early visual processing stages of facial expressions of emotions. In light of this facet of the model, an important prediction is that individuals with congenital facial paralysis (and an altered 
sensorimotor simulation) will present a poorer performance in tasks that investigate in a targeted way the quality of VWM representations of facial expressions of emotions.

In the present study we used an experimental paradigm defined Delayed Estimation Task (DET; Zhang and Luck, 2008) to investigate the precision with which face representations are maintained in VWM. To note, previous studies have used DET to study the precision of VWM representations of faces (Krill et al., 2018; Yao et al., 2018). In the present investigation, the focus was on the precision of VWM representations of faces with emotional expressions (i.e., happy, angry and fearful) and Moebius and control participants had to select in a test array of face stimuli the correct intensity of a facial expression presented about 1 second earlier (i.e., the to-be-memorized face stimulus).

The DET performance, and therefore the precision of face representations in VWM, was assessed as the deviation from the correct response in terms of the to-be-memorized Facial Expression Level (i.e., from 0 to 7 where 0 corresponded to a correct response and the values progressively increasing to a progressively increasing error in terms of precision). The model with the best combination of predictors was selected based on the Akaike Information Criteria (AIC; Akaike, 1974) and contained the Group factor, the Emotion factor and the interaction between Emotion and Facial Expression Level. The effect of the Emotion indicated that participants, on the whole, tended to maintain less precise representations of faces with an expression of fear compared to faces with expressions of anger and happiness. The interaction between Emotion and the Facial Expression Level indicated instead that errors tended to increase as the intensity for fearful expressions increased and tended to decrease as the intensity of happy expressions increased. Instead, for angry expressions, there was no effect related to the level of intensity of the emotion. 
For the purposes of this investigation, the most relevant result refers to the Group effect which indicated a worse performance in terms of precision of VWM representations of emotional expressions for Moebius participants compared to healthy participants. Moebius' performance was worse than that of healthy control subjects for all the three facial expressions tested. These results are in line with the experimental predicted outcomes and support the hypothesis that a congenital alteration of the sensorimotor simulation mechanism could compromise relatively early visual stages of processing of facial expressions of emotions. These findings, moreover, converge with the recent evidence provided by our research group (Sessa et al., 2018) of a reduction in the amplitude of the SPCN ERP component (i.e., index of precision of VWM representations) for emotional facial expressions (i.e. anger) when participants' facial mimicry was blocked/altered by a constricting gel compared to when their mimicry was free; in particular this modulation of the SPCN amplitude was particularly evident for participants with high levels of empathy.

The present results support the notion that in a population with congenital facial paralysis - such as that of individuals with Moebius syndrome - the impossibility of constructing appropriate sensorimotor representations of facial expressions of others' emotions and of reactivating them during the vision of this type of stimuli may cause a deficit, albeit slight, in the precision with which facial expressions are processed.

Other studies, using labeling paradigms, have reported that Moebius children show deficits not only in autonomic responses to emotional stimuli but also in categorizing facial expressions (Nicolini et al. 2019; De Stefani et al. 2019), as predicted by sensorimotor simulation models. The conclusions of our and other studies seem to contrast with two previous studies in which either a deficit of recognition/discrimination of facial expressions of emotions was not observed in Moebius participants (Bogart and Matsumoto, 2010) or, using a single- 
case analysis approach, it was observed that few Moebius patients show normotypical responses(Vannuscorps et al., 2020) despite at population level the deficits are clearly present. Such apparent discrepancies could be partly attributed to differences in methodologies, where the sensitivity of the task may overshadow the actual difficulties in emotion recognition associated with the syndrome.

In the current study, we therefore further implemented a very sensitive paradigm that tests the precision of facial expression representations (by using expressions also particularly subtle) rather than the ability to categorize between facial expressions of emotions (Bogart and Matsumoto, 2010; Vannuscorps et al., 2020). Furthermore, from a neuroconstructivist perspective, we believe it is highly probable that some rare patients with Moebius syndrome can show normotypical performances (see Vannuscorps et al., 2020) that clearly only a singlecase analyses approach can highlight. However, we do not believe that the observation of these 'normotypical' cases can refute the most recent simulation models that consider sensorimotor simulation as one of the components of the complex architecture underlying emotion processing (see Wood, Rychlowska, et al., 2016) leaving the possibility that in functional reorganization processes, other components of the cognitive and neural architecture may successfully support emotional processing, especially when the processing required by the task at hand concerns levels of analysis not particularly fine-grained (as instead necessary to construct highly precise VWM representations).

One possible objection to the present findings is that the reduced performance of subjects with Moebius syndrome in DET for emotional expressions may depend on a more general visuo-cognitive impairment that is not specific to emotional expressions. However, this interpretation seems unlikely given the absence of group differences in reaction times. Also another study employing a labeling task showed that Moebius children and controls show 
similar latency response (De Stefani et al. 2019) thus supporting the idea that general visuocognitive impairments are not present in these patients. We cannot currently rule out this potential alternative explanation of our results. Future studies should compare the performance of Moebius patients and that of control subjects in standard VWM tasks which include stimuli other than emotional facial expressions, such as colored squares (e.g., Vogel and Machizawa, 2004) or random polygons (e.g., Luria et al., 2010). However, it is useful to note that solid evidence exists that recognition of objects (including faces) is independent of other cognitive abilities (Wilmer et al., 2010; Shakeshaft and Plomin, 2015; Gignac et al., 2016; Richler et al., 2017), and consequently it is difficult to explain the specific deficit related to facial expression processing by referring to a more global visuo-cognitive deficit.

In relation, therefore, to the sensorimotor simulation models, and to the model of Wood and colleagues (2016) more specifically, we believe that the observation that subjects with congenital facial paralysis show a lower performance than the control subjects in a task aimed at test the precision of emotional facial expression representations, is in support of the hypothesis that the simulation process communicates iteratively with the visual system during even relatively early stages, allowing to 'refine' visual representations. These results are also in line with a recent study that has demonstrated modulations of visual ERP components, i.e. P1 and N170, following the blocking of facial mimicry and as a function of the degree of alexithymia of the participants (Lomoriello et al., 2019) supporting therefore, overall, the crucial function of the sensorimotor system in these early phases of visual analysis. A methodological limitation regards the assumption of a linear relationship between Facial Expression Level (treated as continuous variable) and Memory Errors. This can be considered a parsimonious choice given the amount of available data but future studies could consider a non-linear relationship between these variables to better capture the complex pattern depicted in Figure 3. 
We also recognize the potential limit of the study in terms of low sample size due to the fact that Moebius Syndrome, although it represents an extremely interesting test for simulation models, is a very rare pathological condition. However, we believe that our results should be considered in the light of current evidence in the literature, which, in most cases, suggests a deficit, albeit slight, in patients with Moebius syndrome in recognizing others' emotions. The present work would therefore suggest that this deficit is observable in tasks aimed at measuring the precision of the representations in memory, thus supporting the view that the sensorimotor system plays an important role from the earliest stages of processing of others' facial expressions of emotions. 
VWM deficits in Moebius subjects - Gambarota et al.

\section{Credit authorship contribution statement}

Filippo Gambarota: Methodology, Formal analysis, Data Curation, Visualization, Writing Original Draft

Roy Luria: Methodology, Writing - Review \& Editing

Massimiliano Pastore: Data Curation, Supervision, Formal analysis

Elisa De Stefani: Writing - Review \& Editing

Pier Francesco Ferrari: Conceptualization, Writing - Review \& Editing

Paola Sessa: Conceptualization, Investigation, Data Curation, Supervision, Writing - Original Draft, Writing - Review \& Editing 
VWM deficits in Moebius subjects - Gambarota et al.

\section{Acknowledgments}

We would like to thank patients and their families for their willingness to participate in this research. We also thank the students Noemi Alamia and Chiara Maria De Rienzo for their collaboration in the collection of experimental data. 


\section{Figures captions and tables title}

Figure 1: (A) Experimental paradigm. (B) Example array of experimental stimuli from neutral to full facial expression.

Figure 2. Distributions of memory errors (relative frequencies) according to displayed Emotion and Group.

Figure 3. Means and standard errors of Memory Errors score according to displayed emotions, Group and Facial Expression Level.

Figure 4: Estimated effects of the selected model. Shaded areas and bars represent the 95\% confidence interval. Upper left: the main effect of Group. Points represent the estimated means. Upper right: the relationship between Reaction Times and Memory Errors. Below: Interaction between Facial Expression Level and displayed Emotion.

\section{Table 1:}

- Title: Demographic and clinical characteristics of participants with Moebius syndrome

\section{Table 2:}

- Title: Descriptive statistics of Memory Errors and Reaction Times 


\section{References}

Adolphs, R. (2002). Recognizing emotion from facial expressions: psychological and neurological mechanisms. Behavioral and cognitive neuroscience reviews, 1, 21-62

Adolphs, R., Birmingham, E. (2011). Neural Substrates of Social Perception. In: G. Rhodes, M. Johnson, J. Haxby (eds). Oxford Handbook of Face Perception. OUP Oxford.

Akaike, H. (1974). A new look at the statistical model identification. IEEE transactions on automatic control, 19, 716-23

Baron-Cohen, S., Wheelwright, S. (2004). The empathy quotient: an investigation of adults with Asperger syndrome or high functioning autism, and normal sex differences. Journal of autism and developmental disorders, 34, 163-75

Bate, S., Cook, S.J., Mole, J., et al. (2013). First report of generalized face processing difficulties in möbius sequence. PloS one, 8, e62656

Bays, P.M., Catalao, R.F.G., Husain, M. (2009). The precision of visual working memory is set by allocation of a shared resource. Journal of vision, 9, 7.1-11

Bogart, K., Matsumoto, D. (2010). Facial mimicry is not necessary to recognize emotion: Facial expression recognition by people with Moebius syndrome. Social neuroscience, $\mathbf{5}$, $241-51$

Borgomaneri, S., Bolloni, C., Sessa, P., et al. (2020). Blocking facial mimicry affects recognition of facial and body expressions. PloS one, 15, e0229364

Bruce, V., Young, A. (1986). Understanding face recognition. British journal of psychology, 77 ( Pt 3), 305-27 
Calder, A.J., Keane, J., Cole, J., et al. (2000). Facial expression recognition by people with mobius syndrome. Cognitive neuropsychology, 17, 73-87

Darwin, C. (1872). The Expression of the Emotions in Man and Animals. London, England: John Murray.

Davis, J.D., Winkielman, P., Coulson, S. (2017). Sensorimotor simulation and emotion processing: Impairing facial action increases semantic retrieval demands. Cognitive, affective \& behavioral neuroscience, 17, 652-64

De Stefani, E., Nicolini, Y., Belluardo, M., et al. (2019). Congenital facial palsy and emotion processing: The case of Moebius syndrome. Genes, brain, and behavior, 18, e12548

Dimberg, U. (1982). Facial reactions to facial expressions. Psychophysiology, 19, 643-47

Dimberg, U., Thunberg, M., Elmehed, K. (2000). Unconscious Facial Reactions to Emotional Facial Expressions. Psychological Science, 11, 86-89

Du, Y., Zhang, F., Wang, Y., et al. (2016). Perceptual Learning of Facial Expressions. Vision research, 128, 19-29

Ekman, P. (2003). Darwin, deception, and facial expression. Annals of the New York Academy of Sciences, 1000, 205-21

Gambarota, F., Sessa, P. (2019). Visual Working Memory for Faces and Facial Expressions as a Useful 'Tool' for Understanding Social and Affective Cognition. Frontiers in psychology, 10, 2392

Giannini, A.J., James Giannini, A., Tamulonis, D., et al. (1984). Defective Response to Social Cues in Möbius' Syndrome. The Journal of Nervous and Mental Disease, 172, 174-75 
Gignac, G.E., Shankaralingam, M., Walker, K., et al. (2016). Short-term memory for faces relates to general intelligence moderately. Intelligence, 57, 96-104

Goldman, A.I., Sripada, C.S. (2005). Simulationist models of face-based emotion recognition. Cognition, 94, 193-213

Gray, H.M., Tickle-Degnen, L. (2010). A meta-analysis of performance on emotion recognition tasks in Parkinson's disease. Neuropsychology, 24, 176-91

Haxby, J.V., Hoffman, E.A., Gobbini, M.I. (2000). The distributed human neural system for face perception. Trends in cognitive sciences, 4, 223-33

Hess, U., Fischer, A. (2014). Emotional Mimicry: Why and When We Mimic Emotions. Social and personality psychology compass, 8, 45-57

Ipser, A., Cook, R. (2015). Blocking facial mimicry reduces perceptual sensitivity for facial expressions. Journal of vision, 15, 1376-1376

Jolicœur, P., Dell'Acqua, R., Brisson, B., et al. (2011). Visual spatial attention and visual short-term memory: Electromagnetic explorations of the mind. In: V. Coltheart (ed). Tutorials in Visual Cognition. Routledge.

Kanwisher, N., McDermott, J., Chun, M.M. (1997). The fusiform face area: a module in human extrastriate cortex specialized for face perception. The Journal of neuroscience: the official journal of the Society for Neuroscience, 17, 4302-11

Kanwisher, N., Yovel, G. (2006). The fusiform face area: a cortical region specialized for the perception of faces. Philosophical transactions of the Royal Society of London. Series B, Biological sciences, 361, 2109-28 
Krill, D., Avidan, G., Pertzov, Y. (2018). The Rapid Forgetting of Faces. Frontiers in psychology, 9, 1319

Kumar, D. (1990). Moebius syndrome. Journal of medical genetics, 27, 122-26

Kuznetsova, A., Brockhoff, P., Christensen, R. (2017). lmerTest Package: Tests in Linear Mixed Effects Models. Journal of statistical software, 82, 1-26

Lindsay, R.W., Hadlock, T.A., Cheney, M.L. (2010). Upper lip elongation in Möbius syndrome. Otolaryngology--head and neck surgery: official journal of American Academy of Otolaryngology-Head and Neck Surgery, 142, 286-87

Lomoriello, A.S., Maffei, A., Brigadoi, S., et al. (2019). Altering sensorimotor simulation impacts early stages of facial expression processing depending on individual differences in alexithymic traits. arXiv [q-bio.NC]

Luck, S.J. (2008). Visual short-term memory. In: S. J. Luck, A. Hollingworth (eds). Visual Memory. Oxford University Press, p. 43-85.

Luke, S. G. (2017). Evaluating significance in linear mixed-effects models in R. Behavior Research Methods, 49(4), 1494-1502.

Luria, R., Balaban, H., Awh, E., et al. (2016). The contralateral delay activity as a neural measure of visual working memory. Neuroscience and biobehavioral reviews, 62, 100108

Luria, R., Sessa, P., Gotler, A., et al. (2010). Visual short-term memory capacity for simple and complex objects. Journal of cognitive neuroscience, 22, 496-512

McKone, E., Robbins, R., Others (2011). Are faces special. In: G. Rhodes, A. Calder, M. 
Johnson, et al. (eds). Oxford Handbook of Face Perception. Oxford University Press, p. $149-76$.

Meconi, F., Luria, R., Sessa, P. (2014). Individual differences in anxiety predict neural measures of visual working memory for untrustworthy faces. Social cognitive and affective neuroscience, 9, 1872-79

Nakagawa, S., Johnson, P.C.D., Schielzeth, H. (2017). The coefficient of determination R2 and intra-class correlation coefficient from generalized linear mixed-effects models revisited and expanded. Journal of the Royal Society, Interface / the Royal Society, 14

Nakagawa, S., Schielzeth, H. (2013). A general and simple method for obtainingR2from generalized linear mixed-effects models. Methods in Ecology and Evolution, 4, 133-42

Nicolini, Y., Manini, B., De Stefani, E., et al. (2019). Autonomic Responses to Emotional Stimuli in Children Affected by Facial Palsy: The Case of Moebius Syndrome. Neural plasticity, 2019, 7253768

Oberman, L.M., Winkielman, P., Ramachandran, V.S. (2007). Face to face: blocking facial mimicry can selectively impair recognition of emotional expressions. Social neuroscience, 2, 167-78

Pantic, M., Valstar, M., Rademaker, R., et al. (2005). Web-based database for facial expression analysis. In: 2005 IEEE International Conference on Multimedia and Expo. p. 5 pp. - .

Pedersen, L.K., Maimburg, R.D., Hertz, J.M., et al. (2017). Moebius sequence--a multidisciplinary clinical approach. Orphanet journal of rare diseases, 12, 4

Picciolini, O., Porro, M., Cattaneo, E., et al. (2016). Moebius syndrome: clinical features, 
diagnosis, management and early intervention. Italian journal of pediatrics, 42, 56

Pinheiro, J.C., Bates, D. (2000). Mixed-Effects Models in S and S-PLUS. New York: Springer Science \& Business Media.

Ponari, M., Conson, M., D’Amico, N.P., et al. (2012). Mapping correspondence between facial mimicry and emotion recognition in healthy subjects. Emotion , 12, 1398-1403

Psychology Software Tools, Inc. [E-Prime 3.0]. (2016). Retrieved from https://www.pstnet.com.

Rasmussen, L.K., Rian, O., Korshoej, A.R., et al. (2015). Fatal complications during anaesthesia in Moebius syndrome: a case report and brief discussion of relevant precautions and preoperative assessments. International Journal of Anesthesiology \& Research, 3, 116-18

R Core Team (2020). R: A Language and Environment for Statistical Computing. Vienna, Austria: R Foundation for Statistical Computing.

Richler, J.J., Wilmer, J.B., Gauthier, I. (2017). General object recognition is specific: Evidence from novel and familiar objects. Cognition, 166, 42-55

Rychlowska, M., Cañadas, E., Wood, A., et al. (2014). Blocking mimicry makes true and false smiles look the same. PloS one, 9, e90876

Sessa, P., Dalmaso, M. (2016). Race perception and gaze direction differently impair visual working memory for faces: An event-related potential study. Social neuroscience, 11, 97-107

Sessa, P., Luria, R., Gotler, A., et al. (2011). Interhemispheric ERP asymmetries over inferior parietal cortex reveal differential visual working memory maintenance for fearful versus 
neutral facial identities. Psychophysiology, 48, 187-97

Sessa, P., Schiano Lomoriello, A., Luria, R. (2018). Neural measures of the causal role of observers' facial mimicry on visual working memory for facial expressions. Social cognitive and affective neuroscience, 13, 1281-91

Sessa, P., Tomelleri, S., Luria, R., et al. (2012). Look out for strangers! Sustained neural activity during visual working memory maintenance of other-race faces is modulated by implicit racial prejudice. Social cognitive and affective neuroscience, 7, 314-21

Shakeshaft, N.G., Plomin, R. (2015). Genetic specificity of face recognition. Proceedings of the National Academy of Sciences of the United States of America, 112, 12887-92

Stel, M., van Knippenberg, A. (2008). The role of facial mimicry in the recognition of affect. Psychological science, 19, 984-85

Vannuscorps, G., Andres, M., Caramazza, A. (2020). Efficient recognition of facial expressions does not require motor simulation. eLife, 9

Vogel, E.K., Machizawa, M.G. (2004). Neural activity predicts individual differences in visual working memory capacity. Nature, $\mathbf{4 2 8}, 748-51$

Wagenmakers, E.-J., Farrell, S. (2004). AIC model selection using Akaike weights. Psychonomic bulletin \& review, 11, 192-96

Wilmer, J.B., Germine, L., Chabris, C.F., et al. (2010). Human face recognition ability is specific and highly heritable. Proceedings of the National Academy of Sciences of the United States of America, 107, 5238-41

Wood, A., Lupyan, G., Sherrin, S., et al. (2016). Altering sensorimotor feedback disrupts 
visual discrimination of facial expressions. Psychonomic bulletin \& review, 23, 1150-56

Wood, A., Rychlowska, M., Korb, S., et al. (2016). Fashioning the Face: Sensorimotor Simulation Contributes to Facial Expression Recognition. Trends in cognitive sciences, 20, $227-40$

Yao, N., Chen, S., Qian, M. (2018). Trait anxiety is associated with a decreased visual working memory capacity for faces. Psychiatry research, 270, 474-82

Zhang, W., Luck, S.J. (2008). Discrete fixed-resolution representations in visual working memory. Nature, 453, 233-35 